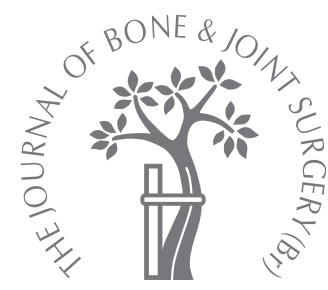

ANNOTATION

\title{
Developing guidelines for venous thromboembolism for The National Institute for Clinical Excellence
}

\author{
INVOLVEMENT OF THE ORTHOPAEDIC SURGICAL PANEL
}

T. Treasure,

L.-Y. Chong,

C. Sharpin,

D. Wonderling,

K. Head,

J. Hill

From National

Clinical Guideline

Centre for Acute and

Chronic Conditions,

London, England
- T. Treasure, MD, MS, FRCS, Professor of Cardiothoracic Surgery

Clinical Operational Research Unit, Department of

Mathematics,

University College, 4 Taviton

Street, London WC1H OBT, UK.

L.-Y. Chong, PhD, Research Fellow

C. Sharpin, BSc, MSc, Senior Information Scientist and Research Fellow

D. Wonderling, BA, MSc,

Health Economics Lead

K. Head, MEng, MSc, Project

Manager/Senior Research

Fellow

J. Hill, BSc, MSc, PhD,

Guidelines Operations Director

National Clinical Guidelines

Centre, Royal College of

Physicians, 1 St. Andrews

Place, Regents Park, London

NW1 4LE, UK.

Correspondence should be sent to Professor T. Treasure; e-mail: tom.treasure@gmail.com

(C2010 British Editorial Society of Bone and Joint Surgery doi:10.1302/0301-620X.92B5. $24448 \$ 2.00$

$J$ Bone Joint Surg $[\mathrm{Br}]$ 2010;92-B:611-16.

Following the publication in $\mathbf{2 0 0 7}$ of the guidelines from the National Institute for Health and Clinical Excellence (NICE) for prophylaxis against venous thromboembolism (VTE) for patients undergoing surgery, concerns were raised by British orthopaedic surgeons as to the appropriateness of the recommendations for their clinical practice. In order to address these concerns NICE and the British Orthopaedic Association agreed to engage a representative panel of orthopaedic surgeons in the process of developing expanded VTE guidelines applicable to all patients admitted to hospital. The functions of this panel were to review the evidence and to consider the applicability and implications in orthopaedic practice in order to advise the main Guideline Development Group in framing recommendations.

The panel considered both direct and indirect evidence of the safety and efficacy, the costeffectiveness of prophylaxis and its implication in clinical practice for orthopaedic patients. We describe the process of selection of the orthopaedic panel, the evidence considered and the contribution of the panel to the latest guidelines from NICE on the prophylaxis against VTE, published in January 2010.

The guidance for prophylaxis against venous thromboembolism (VTE) for patients undergoing surgery published by the National Institute for Clinical Excellence (NICE) in April 2007, raised concerns among British orthopaedic surgeons about the appropriateness of the recommendations in orthopaedics. ${ }^{1,2}$ There were several consistent themes:

1. Contemporary rates of VTE might be lower than those which were used in the economic calculations.

2. Major bleeding in trials might not have captured bleeding at the related surgical site and thus might have underestimated the potential damage to joints and the risk of deep infection.

3. Extrapolation from the reduction of deepvein thrombosis (DVT) to the number of deaths from pulmonary embolism which might have been averted in a larger population was challenged as being unproven.

British orthopaedic surgeons were not alone in their concern about the increasing pressure to give all patients 'potent anticoagulants' ${ }^{3}$ North American orthopaedic surgeons had expressed similar concerns about guidance from the American College of Chest Physicians (ACCP) ${ }^{4}$ and as a result the American Academy of Orthopaedic Surgeons (AAOS) released their own guidance. ${ }^{5}$ In a very public riposte, researchers on thrombosis from McMaster University, Hamilton, Ontario, wrote that "The AAOS panel ignored the randomised data demonstrating that thromboprophylaxis reduces both DVT and PE, and many of their recommendations are based on expert opinion and lack a scientific basis". 6

The debate widened to question who was entitled to give guidance to clinicians. Hirsh and Guyatt" wrote that "clinical experts who write guidelines are often influenced by .... undeclared intellectual conflicts of interest. These conflicts of interest should be managed by placing the final responsibility for recommendations in the hands of unconflicted methodologists". Unfortunately, this rather glossed over the fact that rather than being independent 'methodologists' the authors of the ACCP guidance and its supporters might be perceived by orthopaedic surgeons to represent 'thrombologists' whose professional lives were devoted to the study of VTE and its pharmacological reduction. Orthopaedic surgeons could not rely on them to give appropriate weight to the potential harm caused by 'lesser' bleeding which had not been captured in clinical trials, but was perceived by surgeons to be a very real clinical problem.

Against that background following the NICE 2007 guidance in the United Kingdom, 
representatives of NICE and the British Orthopaedic Association $(\mathrm{BOA})$ had a series of meetings and agreed to engage a representative panel of orthopaedic surgeons fully in the next review of the guidelines. The brief of this panel was to review the evidence presented by the technical team of the National Clinical Guideline Centre and to advise the main Guideline Development Group (GDG) on the implications for clinical practice. The latest guidelines from NICE on the prophylaxis against VTE were published in January $2010 .^{8}$

\section{Methods}

The orthopaedic advisory panel. Applicants were sought in March 2008 to form a panel consisting of orthopaedic surgeons, and a nurse and patient representative to assist in developing guidance on the prevention of VTE for all patients admitted to hospital. This was to include an update of the 2007 guidelines. ${ }^{1,2}$

The initial applications were in writing and preliminary telephone calls from those interested were encouraged. An initial screening was followed by an in-depth telephone interview. The key qualification was to be a practising orthopaedic surgeon, with knowledge of the use of prophylaxis for VTE within the National Health Service, although other applicants who could demonstrate relevant experience were not excluded. Desirable attributes included experience of working in a committee, the ability to develop clinical guidelines, a knowledge of evidence-based medicine and an awareness of the methods used by NICE in developing guidelines. At interview, evidence of communication skills, critical appraisal and team-working were assessed as were personal attributes such as enthusiasm and commitment. It was emphasised that the final decisions had to be made by the GDG, but that the orthopaedic panel would advise to ensure that the evidence and its interpretation were appropriate to clinical practice. A member of the panel was appointed to the GDG. Each time the orthopaedic panel or the GDG met there was disclosure of any conflicts of interests, including personal and non-personal financial and non-pecuniary interests.

Analysis of data. Searches were conducted and the papers evaluated for quality as set out in the NICE Guidelines Manual 2007. ${ }^{9}$ The evidence was reviewed and analysed by the National Clinical Guideline Centre staff, who are an independent group of methodologists commissioned by NICE. Network meta-analysis (NMA) was carried out for the main outcomes: pulmonary embolism, DVT and major bleeding. ${ }^{10}$ This is a statistical technique for combining all direct and indirect evidence from randomised controlled trials (RCTs) into one analysis without breaking randomisation.

Models of cost-effectiveness were developed for different subpopulations. These were based on the NMA results, but also incorporated other outcomes which the panel thought were important to orthopaedic patients. These included the cost of re-operation because of bleeding and the costs and health loss, assessed by the quality-adjusted life-years, due to long-term sequelae such as the post-thrombotic syndrome, chronic thromboembolic pulmonary hypertension and stroke. In the model the relative risk (RR) reductions were applied for DVT overall, symptomatic DVT, non-fatal and fatal pulmonary embolism, post-thrombotic syndrome and chronic thromboembolic pulmonary hypertension. Thus, hypothetically, if a certain strategy were shown to reduce DVT by approximately $60 \%$ then in the model the incidence of fatal pulmonary embolism would also be reduced by $60 \%$. Similarly, the RR increases for major bleeding overall were applied to fatal bleeds and strokes as well as to non-fatal major bleeding.

Sensitivity analyses were used to test the robustness of the results to variations in data in areas of uncertainty, particularly those raised by orthopaedic surgeons. These were also stratified by the baseline risk of pulmonary embolism and the baseline risk of major bleeding, in part to address concerns about the contemporary rates of these outcomes. Population specific baseline risks used in the economic modelling are shown in Table I.

Data presented to the group. Systematically extracted elements of the data from randomised trials were used to construct evidence tables and these, along with the results of the meta-analyses and cost-effectiveness analyses were circulated and presented at the meetings of the orthopaedic panel. The evidence presented was discussed and, in reaching their conclusions, the trade-off between benefits and harms, the costeffectiveness and uncertainties in the data were considered. Recommendations were drafted by the orthopaedic group and discussed further by the main GDG which had the responsibility for the final approval.

\section{Results}

The orthopaedic panel. A panel of seven orthopaedic surgeons, who between them represented surgical practice, was selected. The composition of the panel was checked for balance between the different areas of surgical expertise (Table II). Additionally, a patient representative from the BOA and a nurse specialising in VTE were included.

Elective total hip replacement (THR). The NMA for inhospital prophylaxis for DVT included 46 RCTs linking 16 interventions (Figs. 1 and 2) and 24 RCTs linking 12 interventions for prophylaxis against pulmonary embolism. Trials investigating the use of prophylaxis after discharge from hospital were analysed separately. The risk of major bleeding from pharmacological prophylaxis was determined from an NMA using studies on thromboprophylaxis across all populations, on the basis that the results for specific populations would be imprecise. The analysis included 128 studies describing nine interventions and the patients included those undergoing surgery for a hip fracture in nine studies, hip replacement in 28 , knee replacement in 15 , general medical patients in ten, general surgical patients in 48 , a mix of patients having elective orthopaedic surgery in seven and a mix of patients having any surgery in 11 studies. 
Table I. Population-specific baseline risks (\%) for elective total hip (THR) and total knee replacement (TKR)

\begin{tabular}{|c|c|c|c|}
\hline Baseline risks* & $\%$ THR & $\%$ TKR & Source of data ${ }^{\dagger}$ \\
\hline All-cause morality & 0.7 & 0.5 & At three months, unadjusted for the method of prophylaxis ${ }^{15}$ \\
\hline DVT (symptomatic and asymptomatic) & 45 & 60 & $\begin{array}{l}\text { Meta-analysis of non-prophylaxis arms of THR or TKR RCTs } \\
\text { conducted by NCGC }\end{array}$ \\
\hline Symptomatic PE & 3.4 & Unknown & $\begin{array}{l}\text { Meta-analysis of non-prophylaxis arms of THR or TKR RCTs } \\
\text { conducted by NCGC. Symptomatic PE in TKR patients from } \\
\text { cohort studies ranged from } 0.2 \% \text { to } 1.9 \% \text { and } 1 \% \text { was used as } \\
\text { the baseline risk in cost effectiveness analysis. }\end{array}$ \\
\hline Major bleeding & 1.6 & 1.9 & $\begin{array}{l}\text { Meta-analysis of non-pharmacological prophylaxis arms of } \\
\text { THR or TKR RCTs conducted by NCGC }\end{array}$ \\
\hline
\end{tabular}

Table II. Details of the proportion of the time (\%) currently spent by the seven members of the group in different areas of orthopaedic practice. The last column gives the proportion of time spent by the group as a whole

\begin{tabular}{|c|c|c|c|c|c|c|c|c|}
\hline & SC & NF & SF & PG & DM & DW & CY & All \\
\hline \multicolumn{9}{|l|}{ Upper limb } \\
\hline Shoulder & & & 60 & & & 10 & & 10 \\
\hline Elbow & & & 25 & & & 45 & & 10 \\
\hline Hand & & & & & & 40 & 4 & 6 \\
\hline Peripheral nerve & & & 5 & & & & & $<1$ \\
\hline \multicolumn{9}{|l|}{ Lower limb } \\
\hline Hip & 10 & 7 & & 50 & 7 & & 15 & 13 \\
\hline Knee replacement & 15 & 75 & & 50 & 7 & & 18 & 24 \\
\hline Other knee & & 18 & & & 17 & & & 5 \\
\hline Foot and ankle & & & & & 69 & & 20 & 13 \\
\hline \multicolumn{9}{|l|}{ Other orthopaedic surgery } \\
\hline Fractures and trauma & & & & & & 5 & 43 & 7 \\
\hline Spinal & & & & & & & & 0 \\
\hline Pelvis & & & & & & & & 0 \\
\hline Cancer & 75 & & 5 & & & & & 11 \\
\hline Haemophilia & & & 5 & & & & & $<1$ \\
\hline Total & 100 & 100 & 100 & 100 & 100 & 100 & 100 & 100 \\
\hline
\end{tabular}

The models showed the following:

1. Standard duration of prophylaxis. The use of lowmolecular-weight heparin (LMWH) plus graduated compression stockings in combination was the most clinically effective in reducing pulmonary embolism based on the NMA and was also the most cost-effective. Further analyses showed that a combination of a pharmacological agent (LMWH, unfractionated heparin, or fondaparinux) and mechanical prophylaxis was the most cost-effective strategy for more than $70 \%$ of procedures.

2. Post-discharge prophylaxis. LMWH was more clinically cost-effective than no prophylaxis at all after discharge from hospital.

3. Extended duration prophylaxis. Rivaroxaban was as cost-effective as dabigatran or LMWH.

Elective total knee replacement (TKR). The NMA for DVT included 18 RCTs linking ten interventions. The results of the NMA for DVT in elective knee replacement are shown in Figure 3. There was not enough evidence to complete an NMA for pulmonary embolism. As for THR, the NMA for major bleeding was based on all the groups of patients included in the guidelines. However, the baseline risk of $1.9 \%$ major bleeding obtained from an analysis of the nonpharmacological treatment of patients with a TKR (Table II) was used to calculate absolute risk differences.

Dabigatran and fondaparinux were the most costeffective in the baseline cases and in most of the further analyses, except when discounted costs for drugs were used or when the RRs of major bleeding for dabigatran were assumed to be the same as those for LMWH. ${ }^{8}$ In these circumstances LMWH was the most cost-effective.

\section{Discussion}

The first concern expressed in the introduction was that the quoted number of deaths attributable to VTE was too high. The common source was a House of Commons Select Committee report for 2004 to $2005^{11}$ which states:

"Each year over 25000 people in England die from venous thromboembolism (VTE) contracted in hospital. This is more than the combined total of deaths from breast 


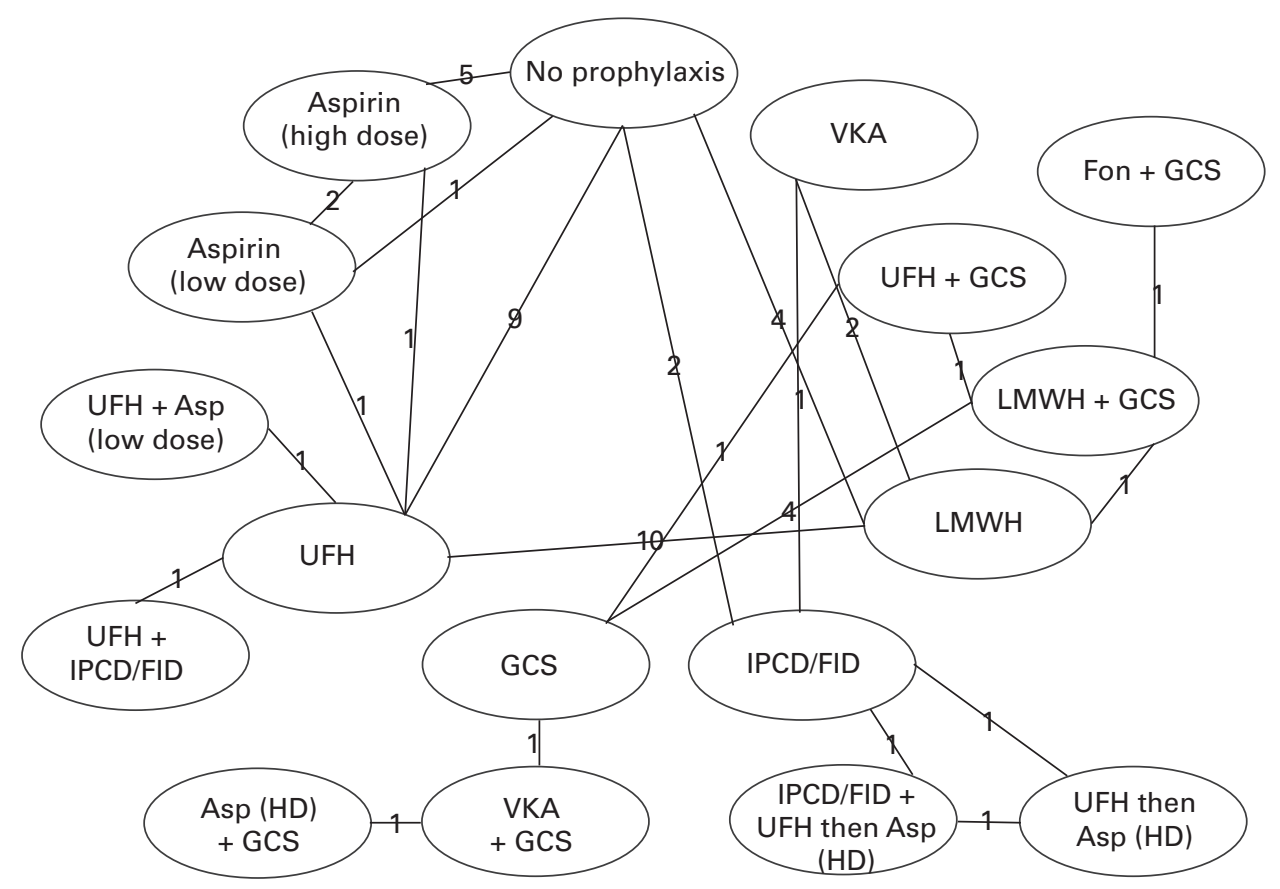

Fig. 1

Network diagram for deep-vein thrombosis as the outcome in total hip replacement. The numbers indicate the number of studies which contributed results for each comparison. The data were extracted from 46 trials and four of these compared three regimes (Asp, aspirin; FID, foot impulse device; Fon, fondaparinux; GCC, graduated compression stockings; HD, high dose; IPCD, intermittent pneumatic compression device; LMWH, low molecular weight heparin; UFH, unfractioned heparin; VKA, vitamin K antagonists).

cancer, AIDS and traffic accidents, and more than twentyfive times the number who die from MRSA. The figures are alarmingly high. Even more alarming is the fact that many of these deaths are preventable. There is a safe, efficacious and cost effective method of preventing venous thrombosis which is not being as widely administered as it should be".

These figures have been challenged, but the widely-held view that too many deaths occur from hospital-acquired VTE has remained. The haematological experts believe that if anything, they are an underestimate of the present situation with respect to hospital-acquired VTE. ${ }^{12,13}$ However, the data used were for all hospital patients, and we are not sure of the exact contribution made by British orthopaedic practice to death rates for VTE. It may be the case that physicians will feel under more scrutiny, than orthopaedic surgeons. Orthopaedic surgeons are very aware of the issue and most use some form of prophylaxis.

Although we did not have recent reliable rates for VTE, the impact of these uncertainties was tested in two-way sensitivity analyses which included a range of values for the baseline risk of major bleeding and pulmonary embolism. These analyses showed that it is cost-effective to provide patients undergoing THR or TKR with prophylaxis for VTE even at lower levels of VTE risk.

The second concern was that the figures quoted did not address the loss of life and limb caused by bleeding related to the use of an anticoagulant. Recent evidence from metaanalysis of RCTs indicates that bleeding is a strong predictor of mortality, ${ }^{14}$ but there are other considerations. With a TKR, bleeding of less magnitude, than might be recorded in trials, could compromise the joint and even the limb. However, there is no firm evidence to support this. Published support will be needed if the risk of balancing VTE and bleeding is to be adjusted. The uncertainties about the impact of bleeding were taken into account in the economic modelling which quantified the potential harms of bleeding. The model had included the cost of re-operation because of bleeding and treatment, and the health loss associated with long-term sequelae such as post-thrombotic syndrome, chronic thromboembolic pulmonary hypertension and stroke.

The third concern was the use of DVT as a 'surrogate' in studies when the major outcome of interest was to avoid death due to VTE. This is a major concern. For example, based on the all-cause mortality rate of $0.7 \%$ as registered in the National Joint Registry, ${ }^{15}$ a study with 20000 patients in each arm would be needed to detect a difference of $10 \%$ in mortality rates. Smaller baseline risks, as in patients with TKR, or smaller reductions in mortality would require increased sample sizes. The first author (TT) participated in a 'think tank' in Washington, DC during 2009 in which this was the central question for a group representing the developers of guidelines for the ACCP, the Federal Food and Drug Administration, international pharmaceutical companies, and North American orthopaedic surgeons. It became evident that the NICE process is more 


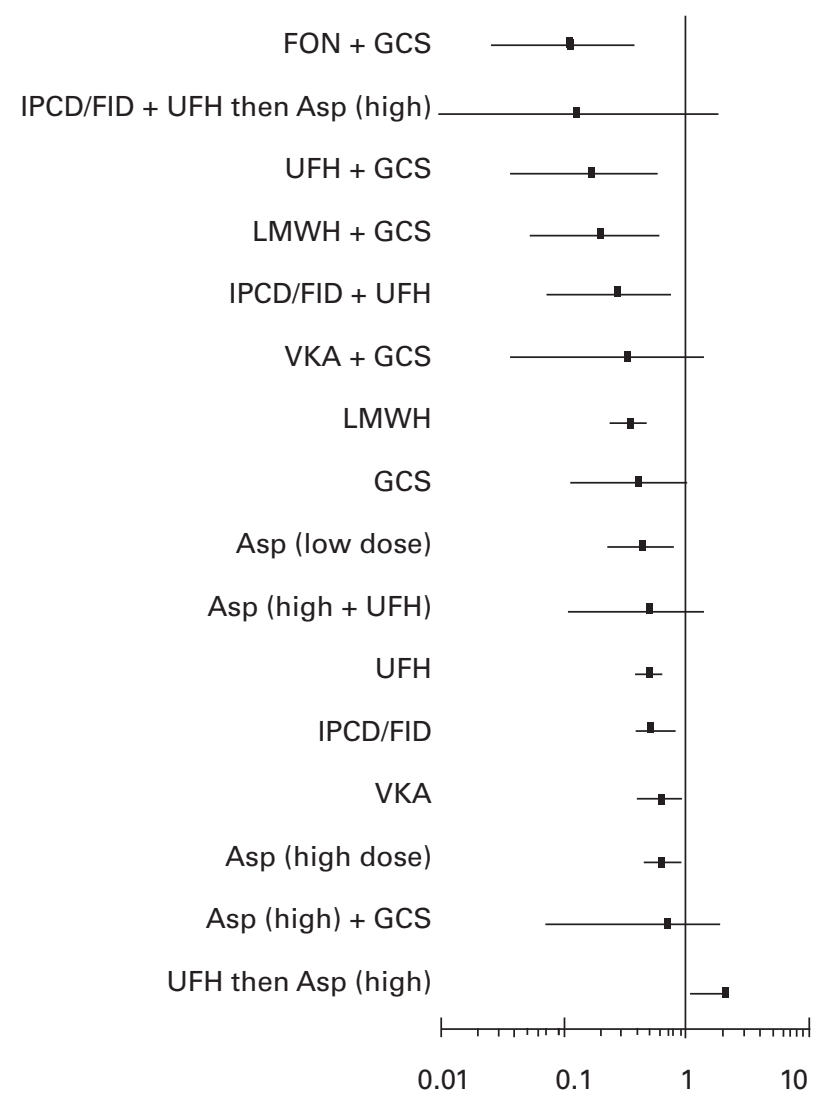

Relative risk

Fig. 2

Diagram showing deep-vein thrombosis as the outcome in total hip replacement. Network meta-analysis results of treatment are compared with no prophylaxis. The dots show the median relative risk and the width of the horizontal lines the $95 \%$ credible intervals (these intervals are the Bayesian equivalent to confidence intervals) (Asp, aspirin; FID, foot impulse device; Fon, fondaparinux; GCC, graduated compression stockings; $\mathrm{HD}$, high dose; IPCD, intermittent pneumatic compression device; LMWH, low molecular weight heparin; UFH, unfractioned heparin; VKA, vitamin $\mathrm{K}$ antagonists).

thorough and more rigorous than any other existing system for the development of guidelines. It was accepted that under most circumstances there is a 'common cause' and reductions in DVT will reduce pulmonary embolism and fatal pulmonary embolism pro rata. This was accepted as a working principle by the orthopaedic panel.

In framing the recommendations, the orthopaedic panel considered the trade-off between clinical benefits and harms, namely, the reduction of all-cause mortality and VTE events versus risk of bleeding. They reviewed the costeffectiveness of the interventions. The quality of evidence providing these data was taken into consideration when making these decisions. It was recognised throughout that the rates of occurrence of bleeding and VTE were too small to be computed on the basis of clinical experience and, apart from bleeding at the surgical site, many clinically important adverse events may lead to admission under

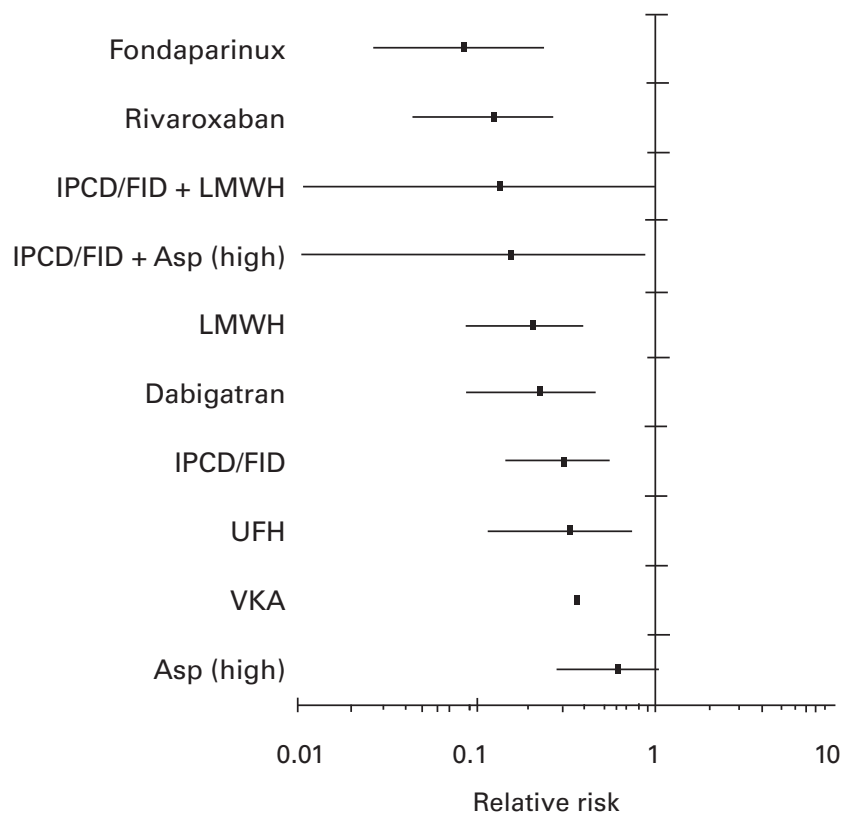

Fig. 3

Diagram showing the network meta-analysis results for deep-vein thrombosis outcome in elective total knee replacement patients - relative risks for each intervention versus no prophylaxis. The dots show the median relative risk and the width of the horizontal lines the $95 \%$ credible intervals (these are the Bayesian equivalent of confidence intervals) (IPCD, intermittent pneumatic compression device; FID, foot impulse device; LMWH, low molecular weight heparin; Asp, aspirin; UFH, unfractioned heparin; VKA, vitamin $\mathrm{K}$ antagonist).

other disciplines. When clinicians are aware of complications affecting individual patients, this may lead to decisions not in the best interest of all subsequent patients. ${ }^{16}$

Despite a large number of studies available, there are still limitations in the availability of clinically important and relevant outcomes to guide the formation of suitable recommendations. Ideally, a reduction in all-cause mortality is the most important outcome in these relatively healthy populations. However, as noted by the orthopaedic panel, the rarity of these events results in very imprecise estimates. Neither the meta-analysis nor individual studies were powered to detect a difference in mortality for any of the interventions and it is unlikely that such studies are available.

We wish to thank the members of the orthopaedic panel: S. Carter, N. Fiddian, S. Frostick. P. Gregg, D. McBride, D. Warwick and C. Young.

No benefits in any form have been received or will be received from a commercial party related directly or indirectly to the subject of this article.

\section{References}

1. No authors listed. Venous thromboembolism: reducing the risk of venous thromboembolism (deep vein thrombosis and pulmonary embolism) in inpatients undergoing surgery. National Institute for Health and Clinical Excellence 2007 http:// www.nice.org.uk/CG46 (date last accessed 2 February 2009).

2. Hill J, Treasure T. Reducing the risk of venous thromboembolism (deep vein thrombosis and pulmonary embolism) in patients having surgery: summary of NICE guidance. BMJ 2007;334:1053-4.

3. Sharrock NE, Gonzalez Della Valle A, Go G, Lyman S, Salvati EA. Potent anticoagulants are associated with a higher all-cause mortality rate after hip and knee arthroplasty. Clin Orthop 2008;466:714-21. 
4. Geerts WH, Bergvist D, Pineo GF,et al. Prevention of venous thromboembolism: American College of Chest Physicians evidence-based clinical practice guidelines (8th edition). Chest 2008;133(6 Suppl):381-453.

5. Haas SB, Barrack RL, Westrich G, Lachiewicz PF. Venous thromboembolic disease after total hip and knee arthroplasty. J Bone Joint Surg [Am]2008;90-A:2764-80.

6. Eikelboom JW, Karthikeyan G, Fagel N, Hirsh J. American Association of Orthopaedic Surgeons and American Collee of Chest Physicians guidelines for venous thromboembolism prevention in hip and knee arthroplasty differ: what are the implications for clinicians and patients? Chest 2009;135:513-20.

7. Hirsh J, Guyatt G. Clinical experts or methodologists to write clinical guidelines? Lancet 2009;374:273-5.

8. No authors lised. Venous thromboembolism: reducing the risk of venous thromboembolism (deep vein thrombosis and pulmonary embolism) in patients admitted to hospital. Methods Evidence \& Guidance. London: National Clinical Guidance Centre - Acute and Chronic Conditions. 2010:CG92. http://www.nice.org.uk/nicemedia/pdf/ CG92FullGuideline.pdf (date last accessed, 17 March 2010).

9. No authors listed. National Institute for Health and Clinical Excellence. The Guidelines Manual. http://www. nice.org.uk/aboutnice/howwework/developingniceclinicalguidelines/clinicalguidelinedevelopmentmethods/theguidelinesmanual2007/ the_guidelines_manual_2007.jsp (date last accessed 1 February 2009).
10. Lu G, Ades AE. Combination of direct and indirect evidence in mixed treatment comparisons. Stat Med 2004;23:3105-24.

11. No authors listed. House of Commons Health Select Committee. The prevention of thromboembolism in hospitalised patients: second report of session 2004-05. House of Commons Health Committee 2005. http://www.publications.parliament.uk/pa/ cm200405/cmselect/cmhealth/99/99.pdf (date last accessed 1 February 2009).

12. Cohen AT, Agnelli G, Anderson FA, et al. Venousthromboembolism (VTE) in Europe: the number of VTE events and associated morbidity and mortality. Thromb Haemost 2007;98:756-64.

13. Hunt BJ. Awareness and politics of venous thromboembolism in the United Kingdom. Arterioscler Thromb Vasc Biol 2008;28:398-9.

14. Eikelboom JW, Quinlan DJ, O'Donnell M. Major bleeding, mortality, and efficacy of fondaparinux in venous thromboembolism prevention trials. Circulation 2009;120:2006-11.

15. Choudhry NK, Anderson GM, Laupacis A, et al. Impact of adverse events on prescribing warfarin in patients with atrial fibrillation: matched pair analysis. BMJ 2006;332:141-5.

16. No authors listed, National Joint Registry. National Joint Registry 2010. http:// www.njrcentre.org.uk/njrcentre/default.aspx (date last accessed 1 February 2009). 